\title{
Performance Evaluation of Protein Sequence Clustering Tools
}

\author{
Haifeng Liu and Loo-Nin Teow \\ DSO National Laboratories (Kent Ridge), 27 Medical Drive, Singapore 117510 \\ lhaifeng@dso.org.sg
}

\begin{abstract}
This paper aims to evaluate the clustering quality of various protein clustering tools that are publicly available as standalone applications. We first review the current protein sequence clustering methods, and introduce a new incrementally clustering tool denoted as PINC. We then propose an intuitive performance metric for evaluating them. The evaluation results of the tools on the public database Pfam are reported.
\end{abstract}

\section{Introduction}

With the enormous growth of public sequence database, grouping the sequences into protein families, is increasingly becoming important as it provides evolutionary, functional and structural information on the sequences. Protein families can be defined as those groups of molecules which share significant sequence similarity ([7]). Well characterized proteins within a family can hence allow one to reliably assign functions to family members whose functions are not known or not well understood. Many methods have been developed for clustering protein sequences according to similarity (or distance) information. This approach usually groups homologous proteins together via a similarity measure obtained by sequence comparison. However, correctly clustering a large number of protein sequences based on the approach still remains a huge challenge due to the following inherent characteristics of proteins:

- Multi-domain proteins. Proteins containing multiple domains that may be similar to different sets of unrelated clusters can confound the protein family detection methods and result in the incorrect grouping of proteins into families.

- Promiscuous domains. Promiscuous domains are widespread components of many proteins. Proteins assigned to a protein family purely on the basis of a promiscuous domain probably have very different functions with other members of that family.

- Fragmented proteins. Public databases may contain fragmented protein sequences (peptides). This incomplete information may lead to the incorrect assignment of a protein into a family.

- Distantly related proteins. Certain proteins sharing little sequence identity can have the same function. It is hard to group this kind of distantly related proteins together without comparison of their three-dimensional structures. 
Although progress has been made on computational protein clustering in terms of accuracy and efficiency, very little work in the literature addresses the systematic comparison and evaluation of clustering results from different tools. Typically, different clustering algorithms yield different clustering solutions on the same data, and there is no agreed-upon guideline for choosing among them. In this paper, we first review the current protein sequence clustering methods, and introduce a new incrementally clustering tool denoted as PINC. We then conduct a performance assessment of PINC and selected representatives from a variety of computational protein clustering approaches on unified data sets using an intuitive performance metric proposed by us. The work would provide researchers a good understanding of current protein clustering tools, and assist the application of them in identifying structural and evolutionary relationships between proteins.

\section{Clustering Methods}

Current commonly employed protein clustering methods require sequence similarity relationships as input, but differ in dealing with the following major issues:

1. Domain decomposition. Basically, the protein clustering methods can be divided into two categories: the methods that have a preliminary decomposition of each protein into its constituent domains such as applied in [6, 9, 16, and the methods that directly cluster the protein sequence and are not domain aware including [1, 5, 8, 14 and so on. Although the former methods have achieved success in constructing well-known databases like PRODOM ([6]), ADDA ([12]) and so on, they hampered to a large extent by promiscuous domains, fragmented proteins, and proteins with complex domain structure. This paper focuses on the comparison of the latter methods.

2. Sequence comparison manner. Typically, most of algorithms such as [8, 9, 16, 12, 5] precompute or read in all pairwise sequence comparison. This all-against-all precomputation suffers from an excessive and unpredictable sequence comparison steps for large scale detection of protein families. On the other hand, several incremental clustering algorithms ([11,14]) reduce the number of sequence comparisons by setting a representative sequence for each cluster. To assign a protein sequence into the existing clusters, the sequence is only compared to the representatives of the clusters.

3. Sequence similarity measure. As proposed in [14, the similarity between two protein sequences is first measured by the percentage of identical residues between them whereas most methods including [9, 8, 16, 5] measure the similarity between two protein sequence by the scores obtained from similarity search algorithms such as BLAST and FASTA ([17]).

4. Cutoff threshold. Almost all clustering algorithms set a cutoff threshold to adjust the granularity (or "tightness") of resulted clusters. Usually, the sequence similarity measures are directly adopted as the cutoff threshold 
variables, such as E-value from BLAST ([16]) and FASTA score ([12]). Unfortunately, there is no single cutoff score that works for all families and different families may be formed at different cutoff scores. Other clustering criteria are adopted to solve the problem, such as in ([8]) the power coefficient of a stochastic matrix is varied to control the tightness of clusters. Recently a metric, called cluster utility, that combines inter-cluster dissimilarity and intra-cluster similarity,e has also been proposed to guide the sequence clustering $([13)$.

5. Transitivity. Transitivity relationship is used by those clustering algorithms that precompute sequence comparison to infer distantly related proteins. However it is not fully understood if transitivity always holds and whether transitivity can be extended infinitely ([5]). Typically, a multidomain protein may connect two clearly unrelated proteins and assign them into the same family. In [12, the graph theory based algorithms avoid the problem by controlling specificity of clusters using criteria which set the shared regions among family members. MCL algorithm in [8] simulates random walks withing a graph representing sequence similarity and iteratively eliminates the inter-family similarities due to multi-domain proteins.

In this work, we only conduct the performance evaluation to the programs that are publicly available as standalone applications. We briefly introduce them as follows: (we focus on the methods that automatically clustering the proteins without the detection of domains.)

BLASTCLUST. BLASTCLUST ([1]) automatically and systematically clusters proteins based on pairwise matches found using the BLAST algorithm. It finds pairs of sequences that have statistically significant matches and clusters them using single-linkage clustering which puts a sequence into a cluster if the sequence is a neighbor to at least one sequence in the cluster. The neighbor relationship between two sequences is determined by a score density threshold, whose adjustment also incurs the varied output clusters.

TRIBE-MCL. TRIBE-MCL ([8]) is a publicly available tool that aims to rapidly and accurately cluster protein sequences into families. It relies on the Markov cluster algorithm for the assignment of proteins into families based on precomputed all-against-all sequence similarity information using BLAST. The inflation value parameter $(I>1)$ of the MCL algorithm is used to control the granularity of clusters.

CD-HI. CD-HI (14]) uses the same greedy incremental algorithm as in (11]) where a sequence is compared with the existing representatives of clusters. If the similarity with any representative is above a given threshold, it is then included into that cluster. Otherwise a new cluster is created with the sequence as its representative. The program detects similar sequences by counting the number of identical short words between them, which speeds up the clustering process. The output of the program varies according to the identity threshold $(40-90 \%)$. 


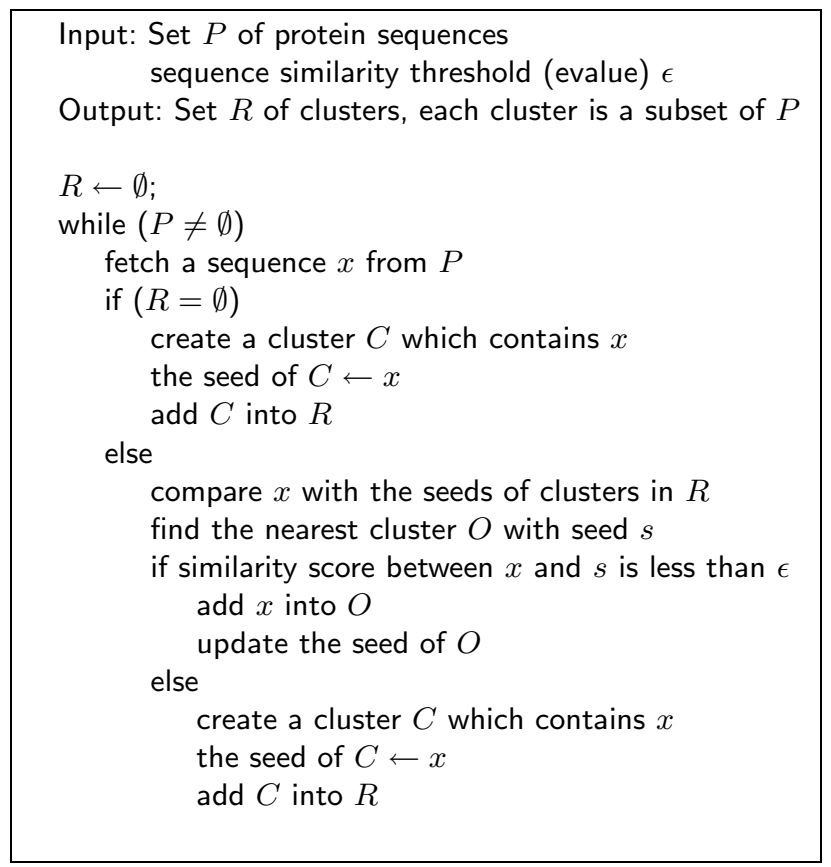

Fig. 1. Clustering algorithm PINC for detecting protein families

PINC. We propose an algorithm, denoted as PINC (Protein INcremental Clustering), which employs the similar incremental clustering algorithm as CD-HI, but uses BLAST to compare sequences as BLAST is a widely used sequence comparison tool and provides statistically significant alignments. The BLAST E-value score is directly used to vary the output of PINC. The details of the algorithm is shown in Fig. 1, whereby the seed of a cluster is a representative of sequences in the cluster. We adopt two options to determine the seed of a cluster. Firstly, to keep the algorithm simple, we determine the longest sequence in a cluster as its seed at present. We denote the algorithm as PINC-L. Alternatively, the seed of a cluster may be the consensus string of a multiple alignment of all sequences in the cluster. This approach, however, may require a large amount of additional computation and hence decrease the effectiveness of the algorithm. In our implementation, we use ClustalW ([19]) to do the multiple sequence alignments. The multiple alignment is updated whenever a new sequence is added into the cluster, either by aligning all the sequences together (version PINC-C1) or incrementally aligning the new added sequence with the existing alignment (version PINC-C2).

\section{Benchmarking Data}

Different protein sequence clustering programs have been evaluated on different data sets. CD-HI was reported to run fast on databases PDB, SWISS-PROT 
(3]) and NR downloaded from the NCBI, but without any mention of its clustering quality. The effectiveness of TRIBE-MCL was tested in an extensive way. It was performed on SWISS-PROT and PDB databases, and the results were then respectively validated using the InterPro protein domain database (2]) and the Structural Classification of Proteins (SCOP) (15) database. 1583 clusters (out of 1821 or $87 \%$ ) obtained by running TRIBE-MCL on SWISS-PROT were found to have full correspondence with domains in InterPro, while $79 \%$ to $87 \%$ of proteins in clusters with SCOP classification are consistent with the SCOP cluster assignment. This validation process is not straightforward when there is a requirement of two or more reference databases being involved. In this paper, we evaluate the clustering tools by testing them on the subsets of Pfam database (release 10.0) of protein families (4]) which contains 6, 190 families and 553, 048 protein sequences. Pfam families were constructed manually or semi-automatically using hidden Markov models (HMMs) based on the proteins in Swiss-Prot (41.10) and TrEMBL (23.15). Pfam families have permanent accession numbers and contain functional annotation and cross-reference to other databases, such as SCOP ([15]), and can be easily downloaded from its public website. The metrics for measuring the quality of clustering programs on the Pfam families are introduced in the next section.

\section{Evaluation Metrics}

To evaluate the comprehensive power of different clustering programs, we have separately applied the programs on the variable number of Pfam families. Given a set of testing protein families, after the clustering operation is performed, we say a family is homogeneous if its members are assigned into one resulted cluster, and a resulted cluster is pure if it only contains the proteins from one testing family. We quantitatively measure the family homogeneity and cluster purity as follows. Let $N_{f}$ be the number of given testing protein families and $N_{c}$ be the number of clusters produced by a tested clustering tool, for a family $j$ having $m_{j}$ members, its family homogeneity, denoted as $F H_{j}$, is defined as $F H_{j}=\sum_{i=1}^{N_{c}}\left(x_{i} / m_{j}\right)^{2}$ where $x_{i}$ is the number of members of the family assigned into cluster $i$. Likewise, for a cluster $i$ containing $n_{i}$ proteins, its cluster purity, denoted as $C P_{i}$, is defined as $C P_{i}=\sum_{j=1}^{N_{f}}\left(y_{j} / n_{i}\right)^{2}$ where $y_{j}$ is the number of members of the family $j$ assigned into the cluster. The above definitions of family homogeneity and cluster purity are based on the complement form of the definition of heterozygosity for genetic markers which is $H=1-\sum p_{i}{ }^{2}([10])$ ). Overall, the family homogeneity (FH) and the cluster purity (CP) obtained by the program are respectively defined as the weighted averages of all individual family homogeneities and all individual cluster purities as below:

$$
F H=\sum_{j=1}^{N_{f}}\left(F H_{j} * m_{j}\right) / \sum_{j=1}^{N_{f}} m_{j}
$$


and

$$
C P=\sum_{i=1}^{N_{c}}\left(C P_{i} * n_{i}\right) / \sum_{i=1}^{N_{c}} n_{i} .
$$

By analogy to the conventional F-measure ([18]) in the field of information retrieval, we measure the overall clustering quality by combining $F H$ and $C P$ as their harmonic mean:

$$
F^{*}=2 * F H * C P /(F H+C P) .
$$

Speed and memory are critical issues when clustering a large number of sequences given limited computer resources. All programs tested in this work run on a PC with dual Pentium III $1 \mathrm{Ghz}$ CPU and 1 gigabyte physical memory which is operated by Red Hat 9.0. We also compare the running times of different programs.

\section{$5 \quad$ Results}

We have firstly run the selected programs on 1,710 protein sequences from 10 randomly chosen Pfam families. The results with different input parameters are shown in Table 1. Clearly, we observe that CD-HI runs very fast but with a poor performance due to a large number of fragmental clusters. This is because the primary goal of CD-HI is to reduce the redundancy of protein database, and it is more suitable for clustering the proteins that share certain sequence identity. The big differences between the $F^{*}$ values obtained by BLASTCLUST with the varied parameter "density threshold $(\mathrm{S})$ " settings imply that we may apply BLASTCLUST only when the input parameter can be carefully determined. PINC-L, PINC-C1 and PINC-C2 (PINCs) are comparable with TRIBE-MCL in terms of the clustering quality, with PINC-C1 achieving the highest $F^{*}$ value of 0.793 when the input E-value threshold is set to 0.1 . In addition, PINC-C1 and PINC-C2 outperform PINC-L by setting the seed of a cluster as the consensus string but at the cost of running speed. In particular, PINC-C1 has a significant drawback in terms of the running speed due to the large amount of multiple sequence alignments involved. Although PINCs run slower than TRIBE-MCL (the running time of TRIBE-MCL includes the time spent on the precomputation of sequence similarities using BLAST), we notice that the number of clusters by TRIBE-MCL is very sensitive to the (inflation) parameter value, whereas PINCs seem to be more robust against different (E-value) parameter values.

As TRIBE-MCL and PINCs outperform the other programs based on the above results, we prefer to utilize them in practice. Before that, we need to further measure their scalability with larger testing data set. We then run TRIBEMCL and PINC-L on 28, 117 protein sequences from 100 randomly chosen Pfam families. (We do not run PINC-C1 and PINC-C2 because they require a few weeks' running time in our PC). The results are shown in Table 2, TRIBEMCL achieves a higher $F^{*}$ value than PINC-L with the specific input parameter 
Table 1. Evaluation results of protein clustering programs running on 10 Pfam families

\begin{tabular}{|c|c|c|c|c|c|c|}
\hline \multicolumn{2}{|c|}{ Tools } & $\begin{array}{c}\text { Number of } \\
\text { clusters }\end{array}$ & $\mathrm{FH}$ & $\mathrm{CP}$ & $F^{*}$ & $\begin{array}{c}\text { Running time } \\
\text { (seconds) }\end{array}$ \\
\hline \multirow{3}{*}{ CD-HI } & $t=0.4$ & 471 & 0.068 & 1 & 0.12 & 4 \\
\cline { 2 - 8 } & $t=0.6$ & 922 & 0.024 & 1 & 0.048 & 2 \\
\cline { 2 - 8 } & $t=0.9$ & 1,695 & 0.006 & 1 & 0.012 & 2 \\
\hline \multirow{3}{*}{ TRIBE-MCL } & $I=1.0$ & 2 & 0.999 & 0.183 & 0.310 & 60,860 \\
\cline { 2 - 8 } & $I=1.5$ & 11 & 0.770 & 0.661 & 0.712 & 530 \\
\cline { 2 - 8 } & $I=2.0$ & 383 & 0.374 & 0.890 & 0.526 & 472 \\
\hline \multirow{3}{*}{ PINC-L } & $e=0.01$ & 52 & 0.467 & 0.997 & 0.636 & 1,527 \\
\cline { 2 - 7 } & $e=0.1$ & 34 & 0.487 & 0.964 & 0.647 & 940 \\
\cline { 2 - 7 } & $e=1$ & 21 & 0.491 & 0.767 & 0.599 & 787 \\
\hline \multirow{3}{*}{ PINC-C1 } & $e=0.01$ & 35 & 0.619 & 0.981 & 0.759 & 41,146 \\
\cline { 2 - 7 } & $e=0.1$ & 26 & 0.668 & 0.976 & 0.793 & 46,163 \\
\cline { 2 - 7 } & $e=1$ & 15 & 0.663 & 0.815 & 0.731 & 48,505 \\
\hline & $e=0.01$ & 45 & 0.494 & 0.990 & 0.659 & 3,064 \\
\cline { 2 - 7 } & $e=0.1$ & 31 & 0.549 & 0.940 & 0.693 & 3,290 \\
\cline { 2 - 7 } & $e=1$ & 15 & 0.601 & 0.776 & 0.678 & 3,726 \\
\hline
\end{tabular}

Table 2. Evaluation results of protein clustering programs running on 100 Pfam families

\begin{tabular}{|c|c|c|c|c|c|c|}
\hline \multicolumn{2}{|c|}{ Tools } & $\begin{array}{c}\text { Number of } \\
\text { clusters }\end{array}$ & FH & CP & $F^{*}$ & $\begin{array}{c}\text { Running time } \\
\text { (seconds) }\end{array}$ \\
\hline \multirow{3}{*}{ TRIBE-MCL } & $I=1.5$ & 325 & 0.587 & 0.909 & 0.713 & 64,792 \\
\cline { 2 - 7 } & $I=2.0$ & 7,448 & 0.195 & 0.952 & 0.324 & 49,180 \\
\hline \multirow{3}{*}{ PINC-L } & $e=0.01$ & 447 & 0.357 & 0.954 & 0.519 & 259,366 \\
\cline { 2 - 7 } & $e=0.1$ & 175 & 0.362 & 0.719 & 0.482 & 104,416 \\
\cline { 2 - 7 } & $e=1$ & 45 & 0.272 & 0.355 & 0.308 & 34,785 \\
\hline
\end{tabular}

$(I=1.5)$ while both programs run quite slowly (TRIBE-MCL requires longer than 2 weeks' running time with $I=1$ ). However, we notice that TRIBE-MCL has a limitation on the input scale because of its precomputation of all-againstall sequence similarity and large amount of memory usage. In fact, TRIBE-MCL runs out of memory and crashes in our PC when we apply it to a clustering of 52, 614 protein sequences from 300 Pfam families, whereas PINC-L achieves a $F^{*}$ value of 0.496 after a run of 3 days. Therefore, for clustering large scale protein sequence sets, TRIBE-MCL should be considered only when abundant computing resources are available, while PINC can give reasonably good clustering accuracies but a long running time with limited computing resources.

\section{Conclusions}

Our evaluation results show that existing tools solely based on sequence similarity between proteins are still deficient in clustering quality and efficiency, 
especially when coping with a large number of proteins, and suggest that more powerful tools need to be developed. In our future work, in order to improve the running speed of PINCs which currently hinders applying PINCs in large scale clustering, we will rewrite the programs in $\mathrm{C}$ language in place of current Perl scripts. In addition, we consider developing a parallel version of PINCs (or other programs) for dealing with the large scale clustering job with parallel computers.

\section{References}

1. S. F. Altschul, T. L. Madden, A. A. Schaffer, J. Zhang, and et al. Gapped BLAST and PSI-BLAST: a new generation of protein database search programs. Nucleic Acids Research, 25(17):3389-3402, 1997.

2. R. Apweiler, T.K. Attwood, A. Bairoch, A. Bateman, E. Birney, and et al. The InterPro database, an integrated documentation resoruce for protein familes, domains and functional sites. Nucleic Acids Research, 29:37-40, 2001.

3. A. Bairoch and R. Apweiler. The SWISS-PROT protein sequence data bank and its supplement TrEMBL in 1999. Nucleic Acids Research, 27:49-54, 1999.

4. A. Bateman, E. Birney, L. Cerruti, R. Durbin., and et al. The Pfam protein familes database. Nucleic Acids Research, 30(1):276-280, 2002.

5. E. Bolten, A. Schlipe, S. Schneckener, D. Schomburg, and R. Schrader. Clustering protein sequences - structure prediction by transitive homology. Bioinformatics, 17(10):935-941, 2001.

6. F. Corpet, F. Servant, J. Gouzy, and D. Kahn. ProDom and ProDom-CG: tools for protein domain analysis and whole genome comparisons. Nucleic Acids Research, 28(1):267-269, 2000.

7. M. O. Dayhoff. The origin and evolution of protein superfamilies. Fed. Proc., 35:2132-2138, 1976.

8. A. J. Enright, S. V Dongen, and C. A. Ouzounis. An efficient alogrithm for largescale detection of protein families. Nucleic Acids Research, 30(7):276-280, 2002.

9. A. J. Enright and C. A. Ouzounis. GeneRAGE: a robust algorithm for sequence clustering and domain detection. Bioinformatics, 16(5):451-457, 2000.

10. H. R. Gregorius. The concept of genetic diversity and its formal relationship to heterozygosity and genetic distance. Math. Biosciences, 41:253-271, 1978.

11. L. Holm and C. Sander. Removing near-neighbour redundancy from large protein sequence collections. Bioinformatics, 14:423-429, 1998.

12. S. Kim. BAG: A graph theoretic sequence clustering algorithm. In http://bio.informatics.indiana.edu/sunkim/BAG, School of Informatics, Indiana University-Bloomington.

13. S. Kim and A. Gopu. Cluster Utility: A new metric to guide sequence clustering. In http://bio.informatics.indiana.edu/sunkim/BAG, School of Informatics, Indiana University-Bloomington.

14. W. Li, L. Jaroszewski, and A. Godzik. Clustering of highly homologous sequences to reduce the size of large protein databases. Bioinformatics, 17(3):282-283, 2001.

15. G. Murzin, A., S. E. Brenner, T. Hubbard, and C. Chothia. SCOP: A structural classification of proteins database for the investigation of sequences and structures. J. Mol. Biol, 247:536-540, 1995.

16. J. Park and S. A. Teichmann. DIVCLUS: an automatic method in the GEANFAMMER package that finds homologous domains in single- and multi-domain proteins. Bioinformatics, 14(2):144-150, 1998. 
17. W. R. Pearson and D.J. Lipman. Improved tools for biological sequence comparison. Proc. Natl. Acad. Sci., 85:2444-2448, 1988.

18. C.J. Van Rijsbergen. Information Retrieval. Butterworths, London, 1979.

19. J.D. Thompson, D.G. Higgins, and T.J. Gibson. CLUSTAL W: improving the sensitivity of progressive multiple sequence alignment through sequence weighting, position-specific gap penalties and weight matrix choice. Nucleic Acids Research, 22(22):4673-4680, 1994. 